\title{
Natural killer cells inhibit breast cancer cell invasion through downregulation of urokinase-type plasminogen activator
}

\author{
HAO JIN* , HYOJEONG CHOI* , EUN-SOOK KIM, HWAN HEE LEE, HYOSUN CHO and AREE MOON \\ Duksung Innovative Drug Center, College of Pharmacy, Duksung Women's University, Seoul 132-714, Republic of Korea
}

Received April 15, 2020; Accepted September 17, 2020

DOI: $10.3892 / o r .2020 .7840$

\begin{abstract}
Triple-negative breast cancer (TNBC) is one of the most aggressive types of breast cancer, and there is no effective therapeutic target to date. Natural killer (NK) cells are functionally diverse lymphocytes that recognize and kill cancer cells. Although it is clear that NK cells exert antitumor activity in the tumor microenvironment, their role in the aggressive progression of TNBC has not been elucidated in detail. In the present study, we investigated the effect of NK cells on MDA-MB-231 TNBC cells using an indirect co-culture system. The invasive phenotype of MDA-MB-231 cells was significantly inhibited by co-culture with NK cells. Notably, the expression of urokinase-type plasminogen activator (uPA) was markedly reduced by NK cells. Cytokine array analysis showed that the levels of interleukin (IL)-10, IL-6, IL-8, C-C motif ligand (CCL)5, and CCL2 were increased in conditioned media from the co-cultured cells. Among these cytokines, IL-6 played a crucial role in the NK cell-induced uPA downregulation and inhibition of the invasive phenotype of MDA-MB-231 cells and Hs578T cells. We analyzed the Gene Expression Profiling Interactive Analysis database for correlations between IL- 6 and uPA with the overall survival of breast cancer patients. The Kaplan-Meier survival analysis revealed that a low IL-6/uPA ratio was associated with the poor survival of breast cancer patients, suggesting it as an important factor for determining the overall survival of breast cancer patients. Taken together, our findings demonstrate that NK cells in the tumor microenvironment inhibit the invasiveness of TNBC cells through the IL-6-mediated inhibition of uPA.
\end{abstract}

Correspondence to: Dr Aree Moon, Duksung Innovative Drug Center, College of Pharmacy, Duksung Women's University, 33, Samyang-ro 144-gil, Dobong-gu, Seoul 132-714, Republic of Korea

E-mail: armoon@duksung.ac.kr

${ }^{*}$ Contributed equally

Key words: natural killer cells, triple-negative breast cancer, urokinase-type plasminogen activator, co-culture system, interleukin-6

\section{Introduction}

Breast cancer occurs with a high incidence in women $(1,2)$ and has an extremely high mortality rate as it has a high likelihood of invading almost all organs, causing metastasis (3). Triple-negative breast cancer (TNBC) is a subtype of breast cancer characterized by the lack of estrogen receptor, progesterone receptor, and human epidermal growth factor receptor 2 expression (4). TNBC cells are highly invasive, spreading to lymph nodes, which often leads to early relapse with distant metastasis (5).

The tumor microenvironment is a complex cellular system that creates an environment in which tumors can become malignant $(6,7)$. Natural killer $(\mathrm{NK})$ cells within the tumor microenvironment have been shown to play an important role in innate immune defense by eliminating tumor cells or in different contexts, pathogen-infected cells, through the production of various cytokines (8-10). Accumulating evidence suggests a role for NK cells in the regulation of cancer metastasis through microenvironmental and systemic processes such as immunosurveillance (11). The function of NK cells depends on the activation or inhibition of receptors on the cell surface, which determines the release of cytotoxic granules and pro-inflammatory cytokines $(12,13)$. Recently, it was shown that NK cells inhibited the migration and invasion of ovarian carcinoma cells (14). The molecular mechanism for the inhibitory effect of NK cells on the invasive phenotype of cancer cells, however, has not been elucidated.

The metastatic spread of tumor cells to distant locations requires invasion and migration, in which matrix-degrading activity is involved (15). Matrix metalloproteinases (MMPs) degrade components of the extracellular matrix (ECM), contributing to cancer cell invasion and metastases $(16,17)$. Our laboratory demonstrated a role for MMP-2 and MMP-9 in the regulation of the invasive phenotype of TNBC cells $(18,19)$. In addition to MMPs, plasmin can degrade ECM components, either directly or indirectly through MMP $(20,21)$. The activated uPA protease cleaves inactive plasminogen to form enzymatically active plasmin, which in turn cleaves proMMP to active MMP $(22,23)$. Accordingly, urokinase-type plasminogen activator (uPA) is involved in multiple physiological and pathologic processes including cell invasion, wound healing, tumor growth, and metastasis (24-27).

In the present study, we examined the effect of NK-92 cells on the invasive phenotype of TNBC cells using an indirect 
co-culture system of NK-92 cells and human TNBC cells. Here we demonstrated an inhibitory effect of NK-92 cells on the invasiveness of TNBC cells. We further showed that uPA downregulation was crucial for the NK-induced inhibition of the invasive phenotype of TNBC cells.

\section{Materials and methods}

Cell culture and reagents. MDA-MB-231 cells were purchased from the Korean Cell Line Bank (KCLB, Seoul, Korea). Human breast carcinoma MDA-MB-231 cells were cultured in RPMI-1640 media (cat. no. 10-041-CVR; Corning Life Sciences) supplemented with $10 \%$ fetal bovine serum (FBS) and $1 \%$ penicillin-streptomycin. Hs578T cells were purchased from KCLB. Human breast carcinoma Hs578T cells were cultured in DMEM media (cat. no. SH30243.01; HyClone; Thermo Fisher Scientific, Inc.) supplemented with 10\% FBS and $1 \%$ penicillin-streptomycin. MCF-7 cells were purchased from the KCLB. Human breast carcinoma MCF-7 cells were cultured in EMEM media [cat. no. 30-2003; American Type Culture Collection (ATCC)] supplemented with $10 \%$ FBS, $1 \%$ penicillin-streptomycin, and $0.01 \mathrm{mg} / \mathrm{ml}$ insulin. Natural killer cell line NK-92 cells were obtained from ATCC. NK-92 cells were cultured in $\alpha$-MEM (cat. no. 32561-037; Gibco; Thermo Fisher Scientific, Inc.) supplemented with $20 \%$ FBS, $1 \%$ penicillin-streptomycin, $0.1 \mathrm{mM}$ 2-mercaptoethanol, and rhIL-2 (200 U/ml). All cells were incubated at $37^{\circ} \mathrm{C}$ in a humidified atmosphere with $5 \% \mathrm{CO}_{2}$.

Indirect co-culture assay. For the indirect co-culture experiments, Transwell inserts were used with a pore size of $0.4-\mu \mathrm{m}$ that can deliver soluble factors but do not allow cell passage. MDA-MB-231 or Hs578T cells were seeded at $5 \times 10^{5}$ cells $/ 6$-well plate and $1 \times 10^{6}$ NK-92 cells were seeded onto the Transwell insert and the plates were incubated for $48 \mathrm{~h}$.

Immunoblot analysis. Whole-cell lysates were prepared using sodium dodecyl sulfate (SDS) lysis buffer. Immunoblot analysis was performed as previously described (28). Primary antibodies to c-Jun (cat. no. sc-74543; dilution 1:1,000), c-Fos (cat. no. sc-52; dilution 1:1,000), p65 NF-kB (cat. no. sc-372; dilution 1:1,000), phospho-p65 NF-kB (cat. no. sc-33020; dilution 1:1,000), interleukin (IL)-6 (cat. no. sc-28343; dilution 1:1,000), C-C motif ligand 2 (CCL2) (cat. no. sc-1304; dilution 1:1,000), uPAR (cat. no. sc-13522; dilution 1:1,000) and mouse anti-goat IgG-HRP (cat. no. sc-2354; dilution 1:3,000) were purchased from Santa Cruz Biotechnology, Inc. Anti-ATF-2 (cat. no. 9226; dilution 1:1,000) and anti-phospho-ATF-2 (cat. no. 9221; dilution 1:1,000) were purchased from Cell Signaling Technology, Inc. Anti-IL-8 (cat. no. ab18672; dilution 1:1,000) was purchased from Abcam, Inc. Anti-uPA (cat. no. MAB1310; dilution 1:1,000) was purchased from R\&D Systems. HRP-conjugated goat anti-mouse (cat. no. 62-6520; dilution 1:3,000) and HRP-conjugated goat anti-rabbit (cat. no. 65-6120; dilution 1:3,000) were purchased from Invitrogen; Thermo Fisher Scientific, Inc. The secondary antibody was attached to fit the primary antibody's origin. The Western Bright ECL kit (Advansta Inc.) was used for band detection. The relative band intensities were determined by quantification of each band using the FluorChem ${ }^{\mathrm{TM}} \mathrm{E}$ (ProteinSimple, Inc.).

Reverse transcriptase (RT)-PCR assay. RNA was extracted from cells using Trizol reagent (Invitrogen; Thermo Fisher Scientific, Inc.) and reverse-transcribed with RT Superscript-III reverse transcriptase (Invitrogen; Thermo Fisher Scientific, Inc.). The primers for uPA (704 bp) were: 5'-AAAATGCTGTGTGCTGCTGACC-3' (forward) and 5'-CCCTGCCCTGAAGTCGTTAGTG-3' (reverse). The primers for IL-10 (500 bp) were: 5'-CTGTGAAAACAAGAG CAAGGC-3' (forward) and 5'-GAAGCTTCTGTTGGCTCC C-3' (reverse). The primers for CCL5 (186 bp) were: 5'-GAG TATTTCTACACCAGTGGCAAG-3' (forward) and 5'-TCC CGAACCCATTTCTTCTCT-3' (reverse). The primers for IL-6 (148 bp) were: 5'-ACTCACCTCTTCAGAACGAAT TG3' (forward) and 5'-CCATCTTTGGAAGGTTCAGGT TG-3' (reverse). The primers for IL-8 (253 bp) were: 5'-GTG GCTCTCTTGGCAGCCTTCCTGAT-3' (forward) and 5'-TCTCCACAACCCTCTGCACCCAGTTT-3' (reverse). The primers for CCL2 (143 bp) were: 5'-GATGCAATCAAT GCCCCAGTC-3' (forward) and 5'-TCCTTGGCCACAATG GTCTTG-3' (reverse). The primers for $\beta$-actin (171 bp) were: 5'-ACTCTTCCAGCCTTCCTTC-3' (forward) and 5'-ATC TCCTTCTGCATCCTGTC-3' (reverse). The same amount of each amplified PCR product was loaded onto 1-2\% agarose gels. Detection was confirmed by Gel Doc ${ }^{\mathrm{TM}} \mathrm{XR}+$ System (Bio-Rad Laboratories, Inc.).

In vitro invasion assay. An in vitro invasion assay was performed as described previously (19). For indirect co-culture assay, MDA-MB-231 cells ( $2 \times 10^{4}$ cells/well) or Hs-578T cells ( $3 \times 10^{4}$ cells/well) were seeded onto the upper compartment of a 24-well Transwell plate. The cells were cultured with NK-92 cell-conditioned media (CM) and incubated $48 \mathrm{~h}$. Media conditioned by NK-92 cells were collected at $48 \mathrm{~h}$. The CM was filtered through $0.22-\mu \mathrm{m}$ pore-size filters and stored at $-70^{\circ} \mathrm{C}$.

Human cytokine antibody array. The human cytokine antibody array kit was purchased from RayBiotech. The cells were cultured in serum-free media for $24 \mathrm{~h}$. Supernatants were collected and centrifuged at $10,000 \mathrm{x}$ g for $10 \mathrm{~min}$ to remove cell debris. The human cytokine array membranes were blocked with blocking buffer for $30 \mathrm{~min}$ at room temperature. $\mathrm{CM}$ was incubated with the array membranes for $1.5 \mathrm{~h}$. After washing, the membranes were incubated with primary antibody for $1.5 \mathrm{~h}$, followed by additional washes, and incubated with a secondary antibody. The membrane-bound proteins were detected using ECL detection reagents (Advansta Inc.). The relative band intensities were quantitated with an Image Analyzer (ProteinSimple).

Casein-plasminogen zymogram assay. The samples was electrophoresed on $10 \%$ SDS-PAGE containing 5\% casein and plasminogen (20 units). After electrophoresis, the gel was washed three times for $30 \mathrm{~min}$ with $2.5 \%$ Triton X-100 solution to remove the SDS and restore the protein. A solution containing $50 \mathrm{mM}$ Tris- $\mathrm{HCl}$ buffer (pH 7.6), $5 \mathrm{mM} \mathrm{CaCl}_{2}$, $0.02 \%$ Brij-35 and $0.2 \%$ sodium azide was added and expressed 
A

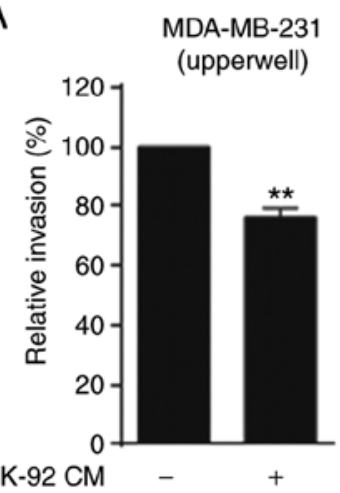

B
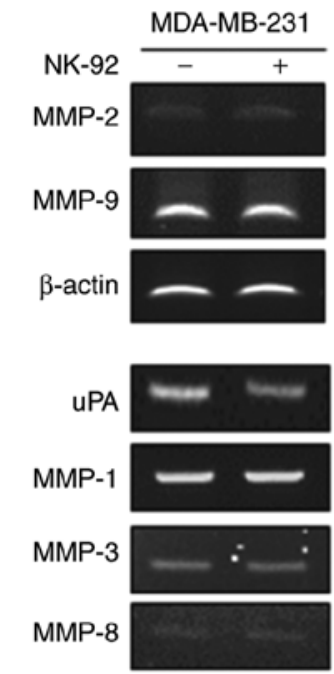

MMP-13

$\beta$-actin
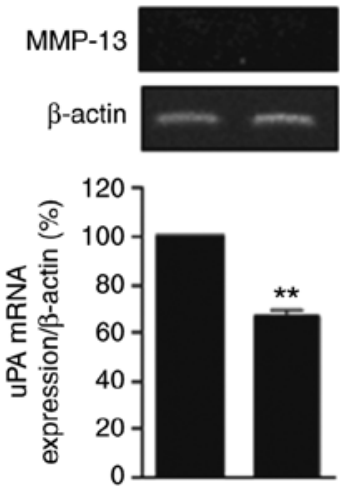

C
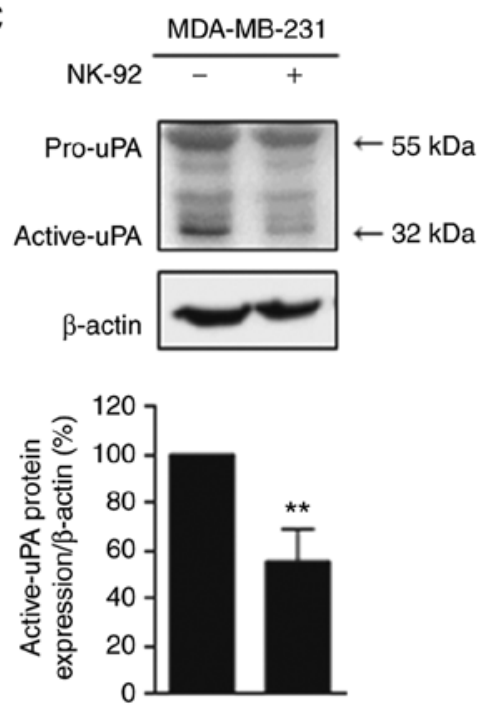

E
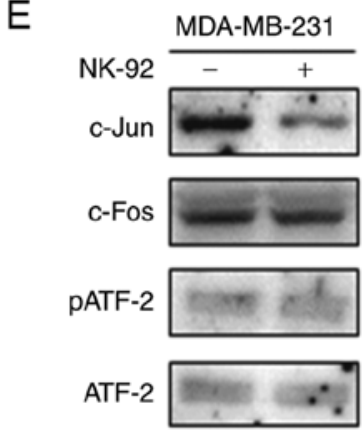

pp65 NF-kB

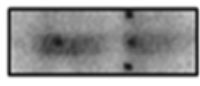

p65 NF-кB

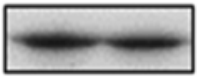

$\beta$-actin
D
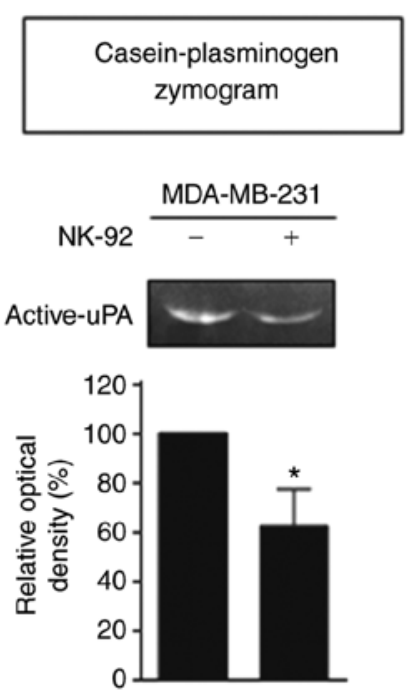

$\mathrm{F}$

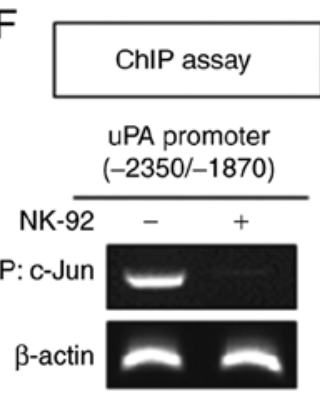

Figure 1. Co-culture with NK-92 cells inhibits the invasive phenotype of MDA-MB-231 cells through uPA downregulation. (A) An in vitro invasion assay was conducted on MDA-MB-231 cells and MDA-MB-231 cells treated with the conditioned media (CM) of NK-92 cells (NK-92 CM) for $48 \mathrm{~h}\left(\mathrm{t}-\mathrm{test}\right.$, ${ }^{* *} \mathrm{P}<0.01$, compared with MDA-MB-231 cells without NK-92 CM). (B) The mRNA levels of MMPs and uPA were detected by RT-PCR analysis in the MDA-MB-231 cells and cells co-cultured with NK-92 cells for $48 \mathrm{~h}$ (t-test, ${ }^{* *} \mathrm{P}<0.01$, compared with MDA-MB-231 cells cultured alone). (C and D) Immunoblot analysis (C) and the casein-plasminogen zymogram assay (D) were performed (t-test, ${ }^{*} \mathrm{P}<0.05$ and ${ }^{* *} \mathrm{P}<0.01$, compared with MDA-MB-231 cells cultured alone, respectively). (E) Immunoblot analysis was performed. (F) ChIP assay was performed using c-Jun primers specific to the AP-1 binding site in the $u P A$ gene promoter. uPA, urokinase-type plasminogen activator; MMPs, matrix metalloproteinases; ATF-2, activating transcription factor 2; p, phosphorylated.

overnight at $37^{\circ} \mathrm{C}$. After staining with $0.5 \%$ Coomassie brilliant blue, the band was observed while decolorizing with $10 \%$ acetic acid.

Chromatin immunoprecipitation (ChIP). The ChIP assay was performed using a Chromatin Immunoprecipitation Assay kit (Upstate Biotechnology Inc.) according to the manufacturer's instructions. The protein-DNA complexes were immunoprecipitated with c-Jun antibodies. Primers specific for the c-Jun binding site in the uPA promoter region were used for DNA amplification as previously described (19).

Ratio of IL-6/uPA and survival analysis of the TCGA/GTEx dataset. The invasive breast carcinoma (BRCA) cancer data sets from 1,070 patients in The GEPIA database were used 

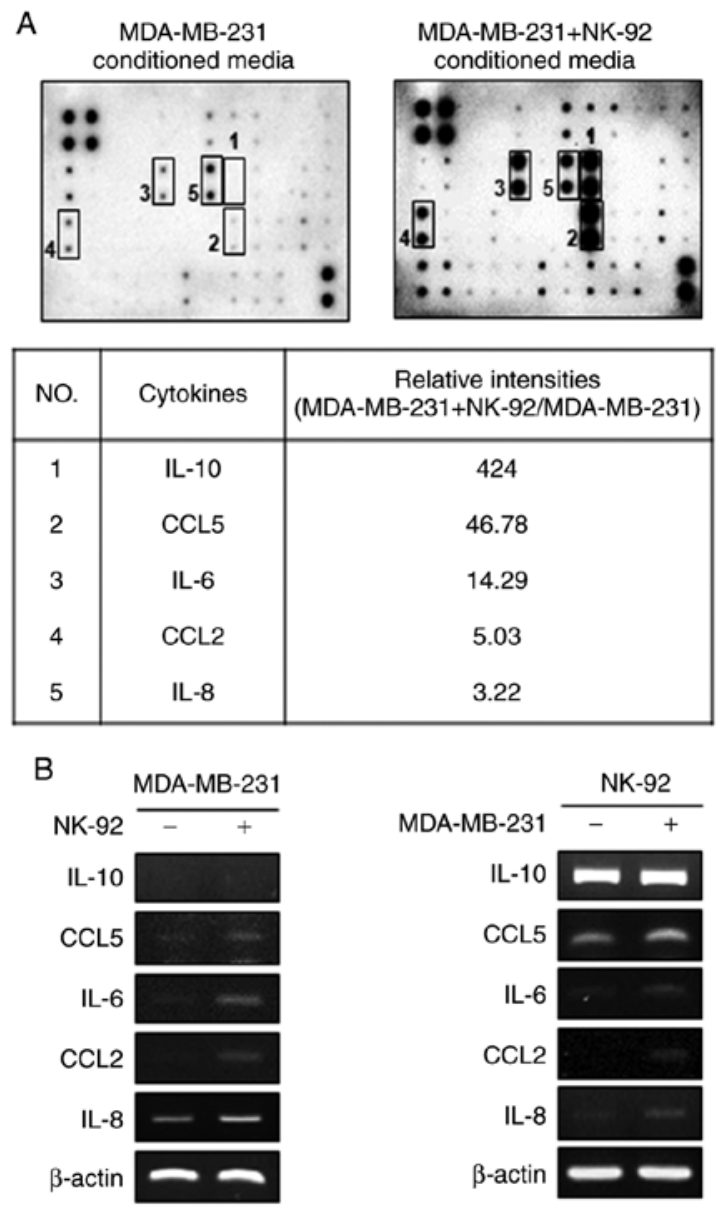

Figure 2. Five cytokines are upregulated by co-culture with NK-92 cells (A) A human cytokine antibody array was conducted on the cultured medium (CM) of mono-cultured MDA-MB-231 cells and that of MDA-MB-231 cells co-cultured with NK-92 cells. Five cytokines with highly relative intensities are listed in the table. (B) RT-PCR analysis was performed to detect the mRNA levels of IL-10, CCL5, IL-6, CCL2, and IL-8. IL, interleukin; CCL, C-C motif ligand.

to explore the correlation of IL-6, uPA, and the IL-6/uPA ratios with survival time in BRCA patients. The ratio of IL-6/uPA in the TCGA/GTEx dataset and its association with tumor stage and overall survival was conducted using the GEPIA database as previously described. Kaplan-Meier survival plots were obtained using the GEPIA online tool (http://gepia.cancer-pku.cn).

Statistical analysis. Statistical significance was analyzed by ANOVA using GraphPad Prism 6 (GraphPad Software, Inc.). Multi-comparison was performed using Tukey's multiple comparisons test. The data are shown as the mean \pm SD from three independent experiments.

\section{Results}

Co-culture with NK-92 cells inhibits the invasiveness of MDA-MB-231 cells through uPA downregulation. To investigate the effect of NK-92 cells on the invasive phenotype of TNBC cells, MDA-MB-231 cells were treated with CM from NK-92 cells. As shown in Fig. 1A, the invasive phenotype of the MDA-MB-231 cells was significantly inhibited by NK-92 cell CM. To identify the proteases involved in the NK cell-induced inhibition of invasion, reverse transcription (RT)-PCR analysis was used to detect matrix-degrading enzymes uPA, MMP-1, MMP-2, MMP-3, MMP-8, MMP-9, and MMP-13 in MDA-MB-231 cells co-cultured with NK-92 cells (Fig. 1B). The mRNA level of uPA was dramatically reduced by co-culture with NK-92 cells, while none of the MMPs were affected. The expression of active uPA (32 kDa) was significantly reduced by co-culture with NK-92 cells as evidenced by immunoblot analysis (Fig. 1C). The caseinplasminogen zymogramassay showed that the matrix-degrading activity of uPA was reduced by co-culture with NK-92 cells (Fig. 1D). These results suggest that the NK-92 cells inhibited the invasive phenotype of MDA-MB-231 cells, possibly via the downregulation of uPA.

To identify the transcription factor(s) responsible for NK-92-induced uPA downregulation, we detected the expression of c-Jun, c-Fos, ATF-2, and p65 NF-kB, which are known uPA transcription factors (29). As shown in Fig. 1E, c-Jun was reduced by co-culture with NK-92 cells, while the others were not affected. The binding of c-Jun to the promoter region of uPA was markedly inhibited by co-culture with NK-92 cells as evidenced by the ChIP assay (Fig. 1F). These data implicate the involvement of c-Jun in the transcriptional regulation of uPA by NK-92 cells.

Secretion of cytokines CCL5, IL-6, CCL2, and IL-8 from NK-92 cells is increased by co-culture with MDA-MB-231 cells. We hypothesized that factor(s) secreted into the CM of co-cultured cells might play a role in the inhibition of invasion and downregulation of uPA in MDA-MB-231 cells. To test this, we conducted a cytokine array and compared the cytokines secreted from mono-cultured MDA-MB-231 cells to cells co-cultured with NK-92 cells. Human cytokine antibody array analysis showed that the levels of IL-6, IL-8, IL-10, and CCL2 and CCL5 were increased in the $\mathrm{CM}$ of co-cultured cells compared to those in the CM of mono-cultured MDA-MB-231 cells (Fig. 2A). The cytokines increased by co-culture with NK-92 cells are listed as a table (Fig. 2A, bottom panel).

Next, RT-PCR analysis was conducted to determine whether these cytokines were secreted from MDA-MB-231 cells or NK-92 cells upon co-culture. The mRNA levels of IL-6, CCL2, and IL-8 were increased in MDA-MB-231 cells co-cultured with NK-92 cells (Fig. 2B, left). The mRNA levels of CCL5, IL-6, CCL2, and IL-8, but not IL-10, were increased in NK-92 cells upon co-culture with MDA-MB-231 cells (Fig. 2B, right). The mRNA levels of tumor necrosis factor (TNF)- $\alpha$ and interferon (IFN) $-\gamma$ were not altered by co-culture (Fig. S1A and B). In addition, the expression levels of IL-6, IL-8, and CCL-2 in NK-92 cells were increased by co-culture with MDA-MB-231 cells (Fig. S1C). The secretion of IL-6, IL-8, and CCL2 from both MDA-MB-231 and NK-92 cells was increased by co-culture.

IL-6 downregulates $U P A$ in MDA-MB-231 cells. To examine the cytokines involved in NK-92-induced uPA downregulation, uPA was analyzed in MDA-MB-231 cells treated individually with CCL5, IL-6, CCL2, or IL-8, whose secretion from NK-92 cells was increased by co-culture. Treatment with recombinant 
A
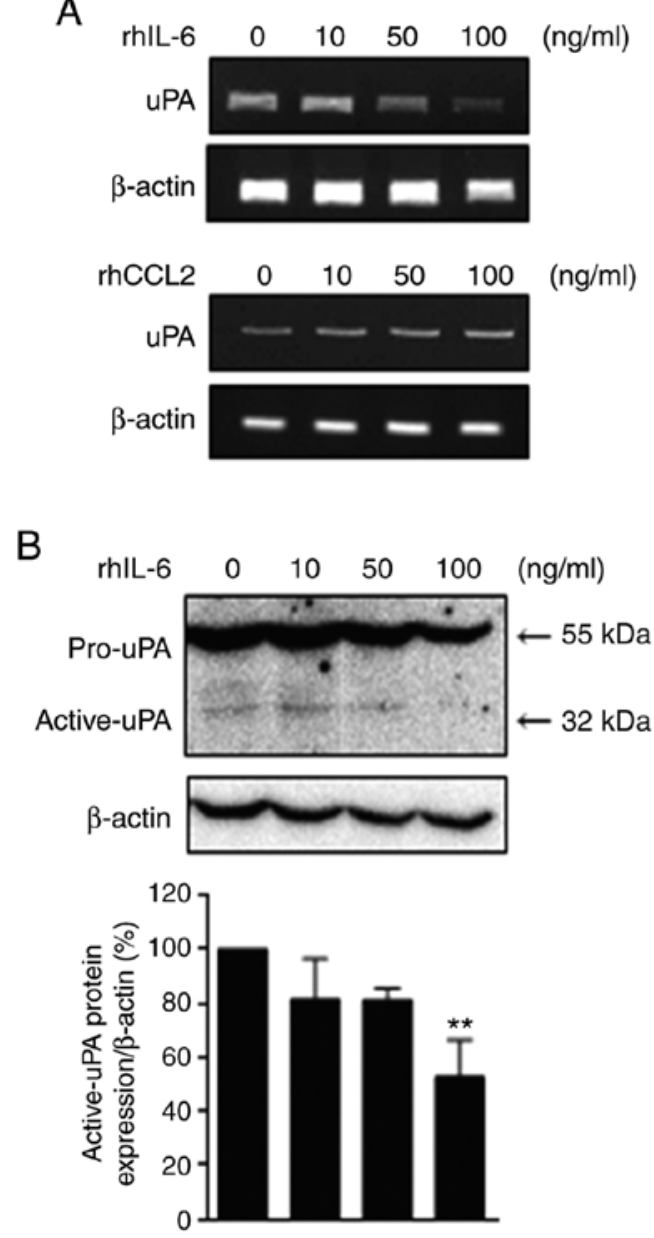

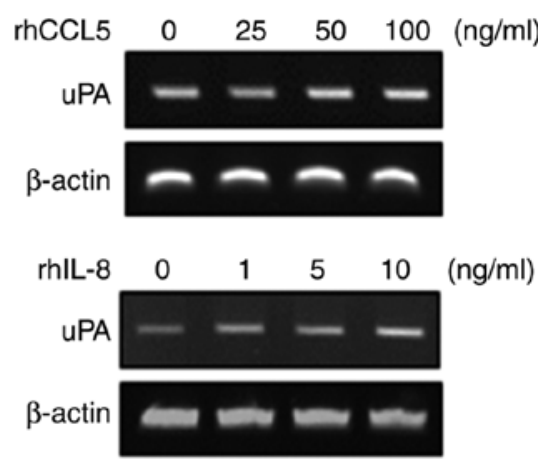

C
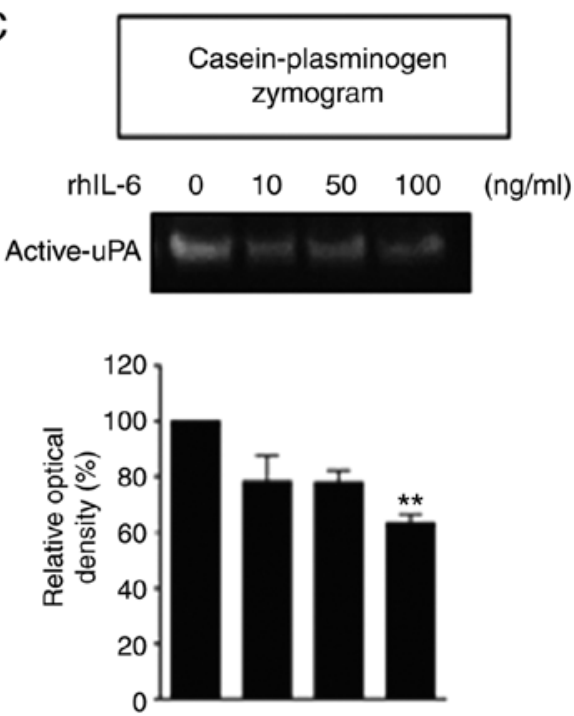

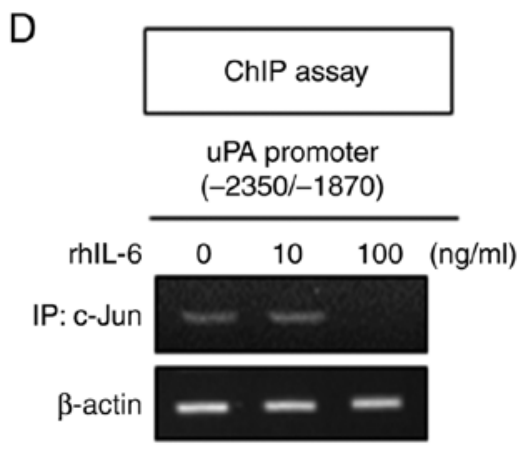

Figure 3. Expression of uPA is reduced by rhIL-6 in MDA-MB-231 cells. (A) The mRNA levels of uPA in MDA-MB-231 cells treated with the indicated concentrations of rhIL-6, rhCCL5, rhCCL2, and rhIL-8 for $48 \mathrm{~h}$ were detected by RT-PCR analysis. (B and C) Immunoblot analysis and the casein-plasminogen zymogram assay were conducted to detect the protein levels (B) and activity (C) of uPA in MDA-MB-231 cells treated with various concentrations of rhIL-6 for $48 \mathrm{~h}$ (one-way ANOVA, ${ }^{* *} \mathrm{P}<0.01$, compared with rhIL-6 $0 \mathrm{ng} / \mathrm{ml}$, respectively). (D) The ChIP assay was performed to detect the binding of the indicated proteins to specific regions of the $u P A$ gene in MDA-MB-231 cells treated with 10 and $100 \mathrm{ng} / \mathrm{ml} \mathrm{rhIL}-6$ for $48 \mathrm{~h}$. rh, recombinant human; uPA, urokinase-type plasminogen activator; IL, interleukin; CCL, C-C motif ligand.

human (rh)IL-6 at concentrations of 50 and $100 \mathrm{ng} / \mathrm{ml}$ reduced the UPA mRNA levels in a concentration-dependent manner (Fig. 3A). Neither rhCCL5, nor rhCCL2 affected the level of uPA mRNA. In contrast, rhIL- 8 increased the level of uPA. Treatment with rhIL-6 decreased uPA protein (Fig. 3B) and activity (Fig. 3C) in a concentration-dependent manner, with a significant inhibition observed at $100 \mathrm{ng} / \mathrm{ml}$. The binding of c-Jun to the uPA gene promoter was inhibited by treatment with rhIL-6 at $100 \mathrm{ng} / \mathrm{ml}$ as evidenced by the ChIP assay (Fig. 3D). These results indicate that a high concentration of
rhIL-6 (100 ng/ml) caused a marked downregulation of uPA in the MDA-MB-231 cells.

IL-6 plays a crucial role in UPA downregulation in TNBC cells. To determine the functional significance of IL-6, we treated the MDA-MB-231 cells with a neutralizing antibody (Ab-IL-6). The reduced expression of uPA by co-culture with NK-92 was significantly increased by the neutralization of IL-6 using Ab-IL-6, both at the mRNA (Fig. 4A) and the protein level (Fig. 4B), as shown by RT-PCR and immunoblot 
A

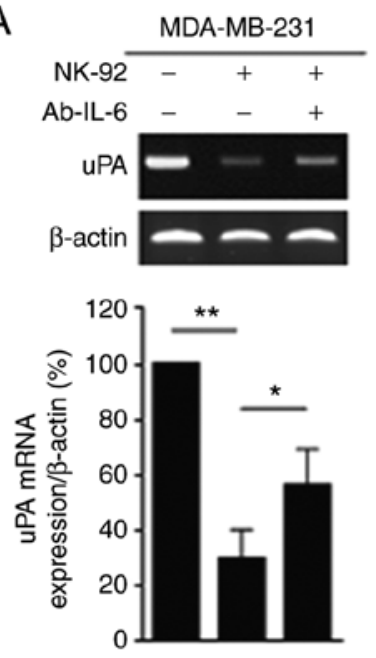

B
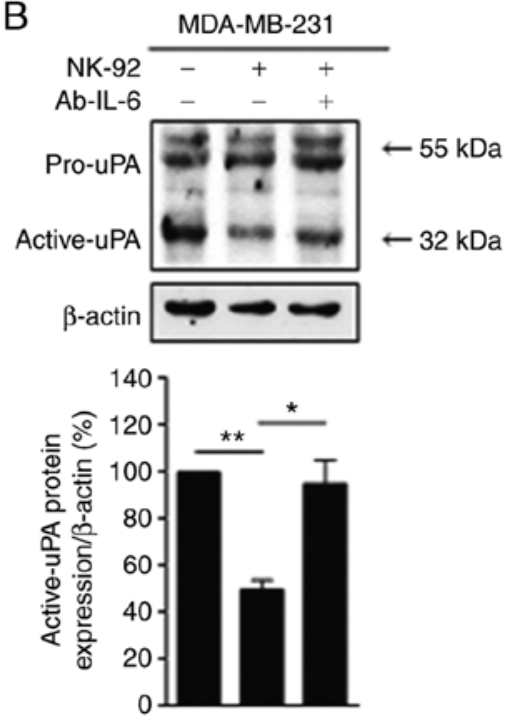

C
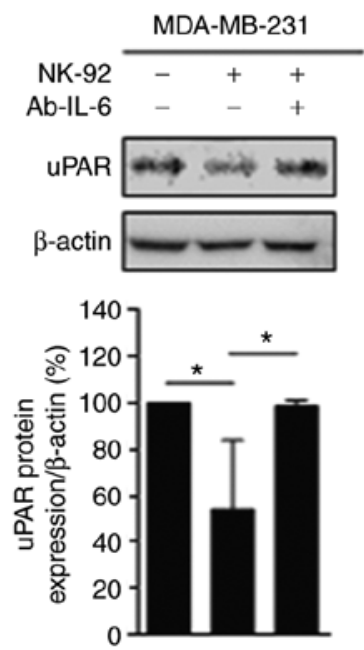

D
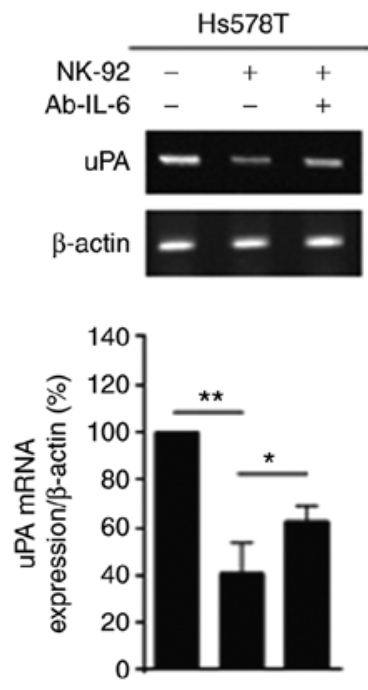

$\mathrm{E}$
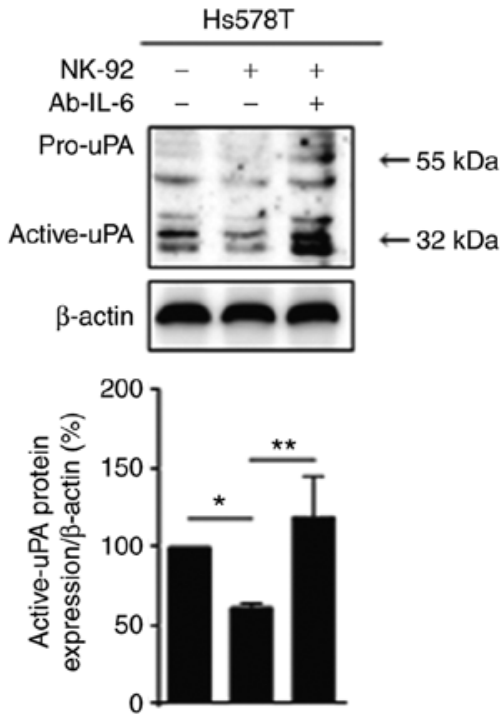

Figure 4. A neutralizing antibody against IL-6 (Ab-IL-6) rescues NK cell-inhibited uPA expression and activity in TNBC cells. (A and B) The expression level of uPA was determined by RT-PCR (A) and immunoblot analysis (B) in mono-cultured MDA-MB-231 cells and MDA-MB-231 cells co-cultured with NK-92 cells (one-way ANOVA, ${ }^{*} \mathrm{P}<0.05$ and ${ }^{* *} \mathrm{P}<0.01$, respectively). The co-cultured cells were treated with Ab-IL-6 (500 ng/ml) for $48 \mathrm{~h}$. (C) The uPAR protein levels were detected by immunoblot analysis in MDA-MB-231 cells and cells co-cultured with NK-92 cells for $48 \mathrm{~h}$ (one-way ANOVA, ${ }^{\circ} \mathrm{P}<0.05$ ). (D and E) RT-PCR and immunoblot analysis were conducted to detect the uPA mRNA (D) and protein levels (E) in Hs578T cells treated Ab-IL-6 for 48 h (one-way ANOVA, ${ }^{*} \mathrm{P}<0.05$ and ${ }^{* *} \mathrm{P}<0.01$, respectively). NK, natural killer; uPA, urokinase-type plasminogen activator; TNBC, triple-negative breast cancer.

analysis, respectively. The downregulation of UPAR protein levels by co-culture with NK-92 was recovered by Ab-IL-6 (Fig. 4C).

We further investigated the inhibitory effect of NK-92 on uPA in another TNBC cell line, the Hs578T cell line. As shown in Fig. 4D and E, co-culture with NK-92 cells inhibited the UPA expression in Hs578T TNBC cells. The reduced uPA mRNA and protein levels by co-culture were recovered by Ab-IL-6 in the Hs578T cells. However, in the MCF-7 cell line, which is a non-TNBC cell line, the UPA mRNA and protein levels were not affected by co-culture with NK-92 cells (Fig. S2A and B). These data suggest that the inhibitory effect of NK-92 on uPA may be specific to TNBC cells.

In addition, treatment with Ab-IL-6 markedly reversed the uPA mRNA and protein levels decreased by rhIL-6 $(100 \mathrm{ng} / \mathrm{ml})$ (Fig. 5A and B, respectively). The effect of IL-6 on uPA was further investigated in Hs578T TNBC cells. As shown in Fig. 5C and D, IL-6 decreased the UPA mRNA and protein levels in Hs578T TNBC cells. These levels were recovered by Ab-IL- 6 in the Hs578T cells. These results demonstrate that IL- 6 secreted by NK-92 cells played a crucial role in the downregulation of uPA in TNBC cells.

A neutralizing antibody against $I L-6$ rescues the NK cell-inhibited invasion in TNBC cells. Next, we investigated the role of IL-6 in regulating the invasive capacity of cells. As shown in Fig. 6A, treatment with Ab-IL-6 significantly restored the MDA-MB-231 cell invasive phenotype that was inhibited by NK-92 cells. The CM of NK-92 cells also inhibited the invasive phenotype of Hs578T TNBC cells, and the decreased invasion was recovered by treatment with Ab-IL-6 (Fig. 6B). In contrast, there was no change in the invasive phenotype 
A
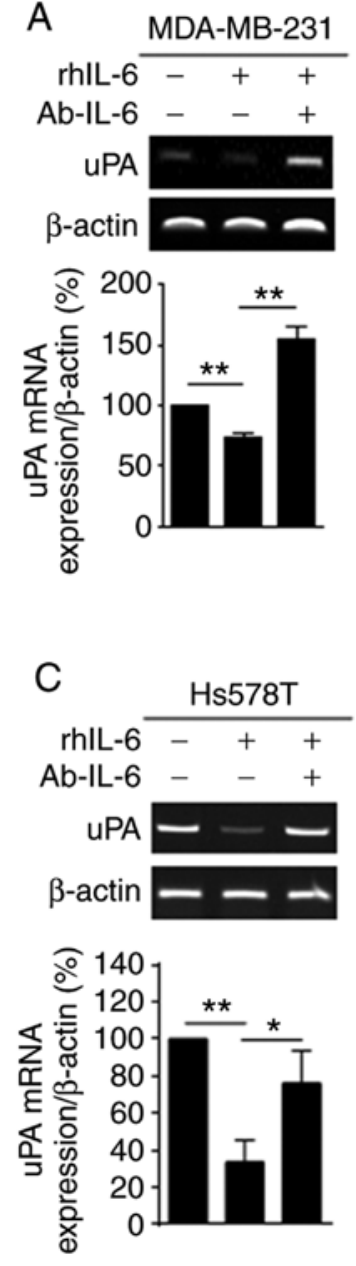

B
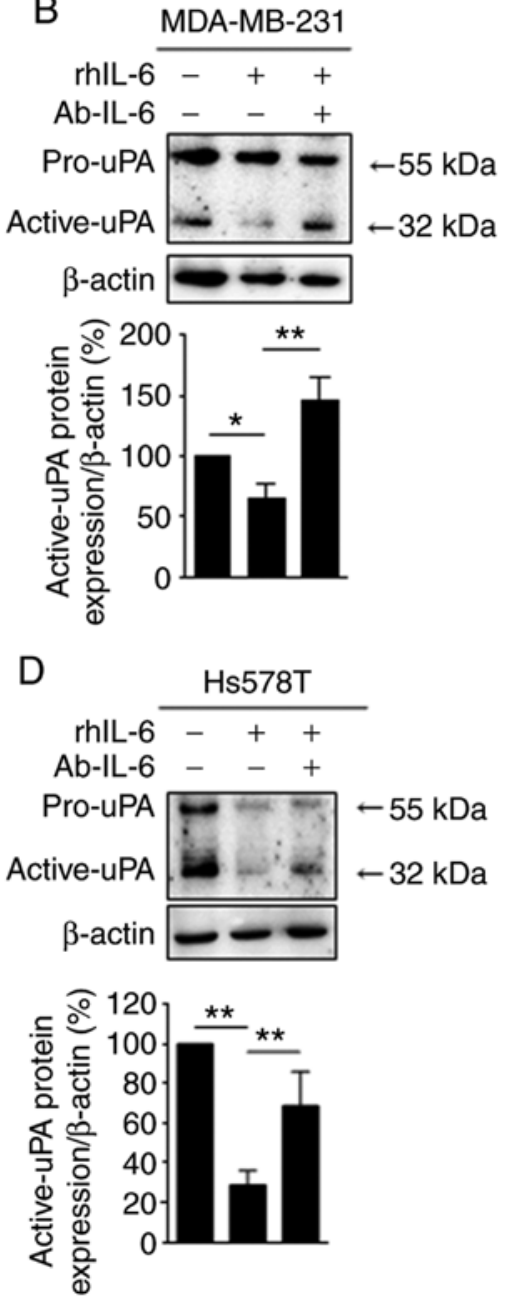

Figure 5. A neutralizing antibody against IL-6 (Ab-IL-6) recovers the rhIL-6-mediated downregulation of uPA expression in TNBC cells. (A and B) RT-PCR (B) and immunoblot analysis (B) were performed on MDA-MB-231 cells treated with rhIL-6 (100 ng/ml) and/or Ab-IL-6 (100 ng/ml) for $48 \mathrm{~h}$ (one-way ANOVA, " $\mathrm{P}<0.05$ and ${ }^{* *} \mathrm{P}<0.01$, respectively). (C and D) The expression levels of uPA following treatment with rhIL-6 and/or Ab-IL-6 in Hs578T cells were determined by RT-PCR (C) and immunoblot analysis (D) (one-way ANOVA, ${ }^{*} \mathrm{P}<0.05$ and ${ }^{* * *} \mathrm{P}<0.01$, respectively). rh, recombinant human; uPA, urokinase-type plasminogen activator; IL, interleukin; TNBC, triple-negative breast cancer.

of MCF-7 cells after co-culture with NK-92 cells (Fig. S2C). These data suggest a TNBC cell-specific inhibition of the invasive phenotype by NK-92 cell CM. When MDA-MB-231 cells were treated with rhIL-6 at $100 \mathrm{ng} / \mathrm{ml}$, the invasive phenotype of MDA-MB-231 cells was significantly inhibited (Fig. 6C). However, treatment with rhIL-6 at lower concentrations did not significantly inhibit invasion. These data suggest that the inhibitory effect of NK-92 cells on TNBC cell invasion may be due to the increased secretion of IL-6, which inhibits uPA expression and activation.

The IL-6/uPA ratio is correlated with the overall survival of breast cancer patients. To examine the clinical relevance of our in vitro data, we analyzed the correlation of IL-6 and uPA with the overall survival of breast cancer patients using the Gene Expression Profiling Interactive Analysis (GEPIA) database (30). The Kaplan-Meier survival analysis revealed that neither IL-6 nor uPA (PLAU) were significantly correlated with overall patient survival (Fig. 6D, left and center, respectively). Of note, a low IL-6/uPA ratio was significantly associated with the poor survival of breast cancer patients, compared to a high ratio $(\mathrm{P}<0.05)$ (Fig. 6D, right). The GEPIA data suggest that the combination of low IL-6 and high uPA may be crucial for malignant breast cancer, suggesting that the ratio of IL- 6 to UPA is a key factor in determining the overall survival of breast cancer patients. Taken together, our findings demonstrated that NK-92 cells downregulated uPA through IL-6, resulting in the inhibition of the invasive phenotype of TNBC cells (Fig. 6E).

\section{Discussion}

Mounting evidence suggests a role of natural killer (NK) cells in the regulation of cancer metastasis, primarily through immunosurveillance by NK cells, which recognizes and kills metastatic cells (11). In the present study, we clearly demonstrated that the invasive phenotype of triple-negative breast cancer (TNBC) cells was significantly inhibited by co-culture with NK-92 cells. Our results suggest that NK-92 cells within the tumor microenvironment not only kill cancer cells via immunosurveillance but can also regulate the metastatic capability of cancer cells. Consistent with our results, a recent 
A

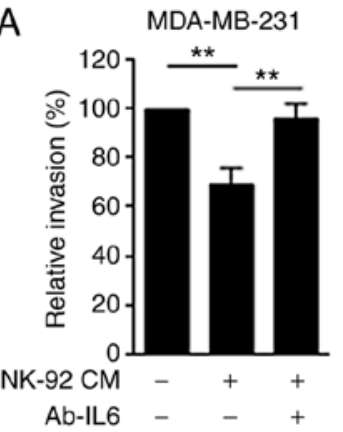

D

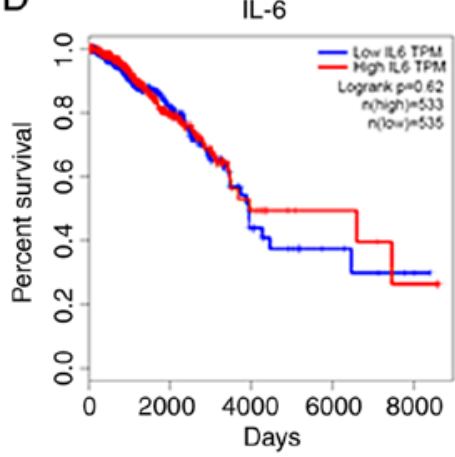

B
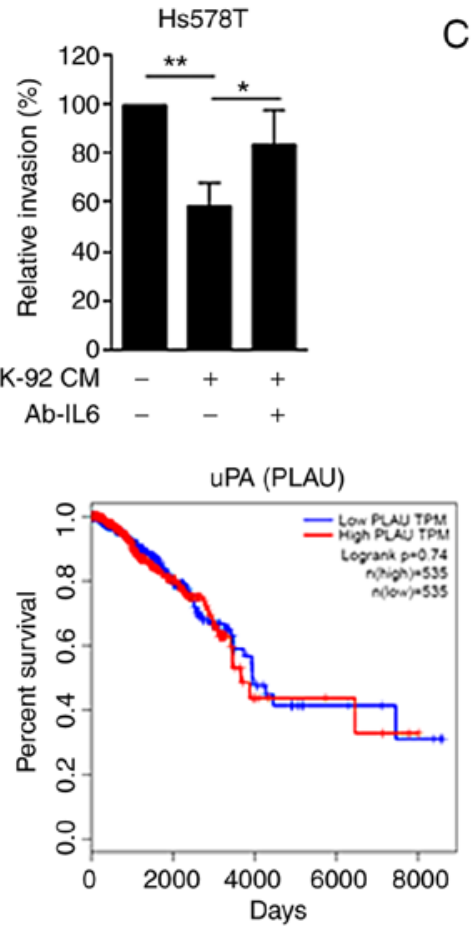

C

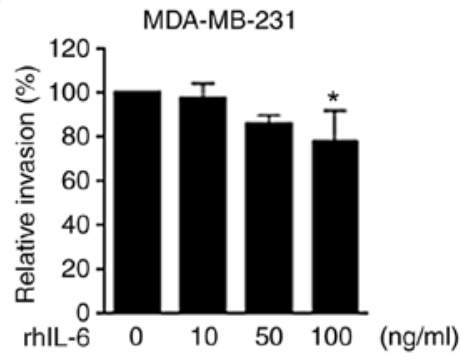

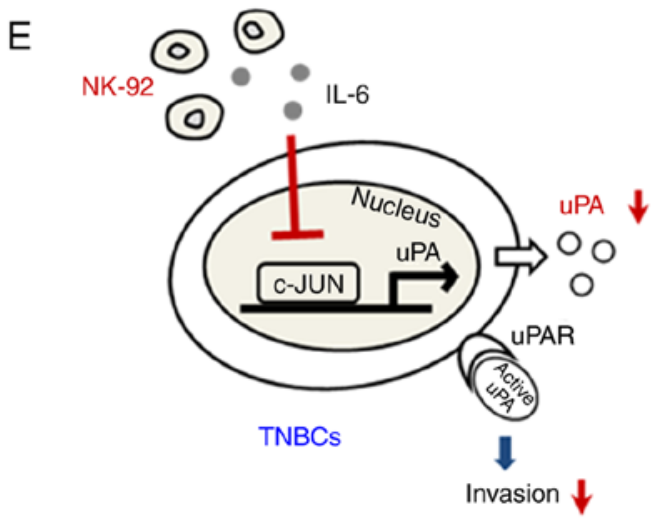

Figure 6. IL-6/uPA is important for the invasiveness of TNBC cells. (A and B) An in vitro invasion assay was conducted on MDA-MB-231 (A) and Hs578T cells (B) treated with a neutralizing antibody against IL-6 (Ab-IL-6) $(500 \mathrm{ng} / \mathrm{ml}$ ) or NK-92 cell-CM (culture media) for $48 \mathrm{~h}$ (one-way ANOVA, "P $<0.05$ and ${ }^{* *} \mathrm{P}<0.01$, respectively). (C) An in vitro invasion assay was performed on MDA-MB-231 cells treated with various concentrations of rhIL-6 for $48 \mathrm{~h}$ (one-way ANOVA, "P<0.05). (D) Kaplan-Meier survival curves showing the probability of survival for patients according to IL-6 (left) and uPA (center) levels, and the IL-6/uPA ratio (right) according to the GEPIA database (http://gepia.cancer-pku.cn). (E) A proposed model for NK-92-mediated uPA downregulation leading to the inhibition of the invasive phenotype of TNBC cells. rh, recombinant human; uPA, urokinase-type plasminogen activator; uPAR, uPA receptor; IL, interleukin; TNBC, triple-negative breast cancer.

paper showed that the migration and invasion of ovarian carcinoma cells were inhibited by NK cells (14).

In an effort to identify matrix-degrading enzymes that could be involved in NK-inhibited TNBC cell invasion, the levels of various matrix metalloproteinases (MMPs) were measured, as well as urokinase-type plasminogen activator (uPA) in MDA-MB-231 cells co-cultured with NK-92 cells. Here, we showed, for the first time, that NK-92 cells downregulated uPA, which plays a crucial role in the inhibition of an invasive phenotype of TNBC cells. A growing body of evidence supports a role for uPA as a prognostic factor in breast cancer (31). High levels of uPA significantly correlate with tumor aggressiveness and poor outcomes in breast cancer $(32,33)$. These results, in conjunction with our findings, suggest that the inhibition of uPA may be able to control the aggressive progression of breast cancer.
NK cell recognition of infected cells or cancer cells stimulates cytokine secretion (34). Among the cytokines secreted from NK-92 cells upon co-culture, interleukin (IL)-6 inhibited the expression and activity of uPA in MDA-MB-231 cells, shown by RT-PCR, immunoblot, and casein-plasminogen zymogram analysis. The ChIP assay showed that IL-6 decreased the binding of c-Jun to the uPA promoter. By using a neutralizing antibody against IL-6, the inhibitory effect of IL-6 on uPA downregulation and invasion in TNBC cells was further confirmed.

Treatment with rhIL-6 at a high concentration $(100 \mathrm{ng} / \mathrm{ml})$ significantly inhibited the invasive phenotype of MDA-MB-231 cells, implying that the increased secretion of IL- 6 by NK-92 cells exerted an inhibitory effect on invasion through uPA downregulation. However, in contrast to our results, elevated levels of IL-6 were shown to be correlated with aggressive tumor growth in several types of cancer, including nasopharyngeal, esophageal, 
and pancreatic cancers (35-37). The reported tumor-promoting role of IL-6 was due mostly to the stimulation of tumor cell proliferation and survival through activation of the PI3K, MEK, and JAK/STAT pathways (38-40). Of note, uPA downregulation was observed at low concentrations of IL-6, whereas the invasive phenotype of the TNBC cells was not inhibited at these concentrations. These results suggest that the inhibitory effect of IL-6 on tumor cell invasion may only be achieved at relatively high concentrations. In support of this hypothesis, the GEPIA analysis showed that a high IL-6/uPA ratio was more advantageous to the overall survival of breast cancer patients. Moreover, the apparent discrepancy between these earlier reports and our findings may also be explained, at least in part, by the fact that our experimental system was a co-culture system with TNBC cells and NK-92 cells, and therefore, lacks other surrounding immune cells, such as $\mathrm{T}$ cells and macrophages. The inhibition of uPA activity by IL-6 may be limited to TNBC cells, but these results have extraordinary significance for breast cancer metastasis and breast cancer treatment.

A growing number of studies have attempted to target the uPA-uPAR system to suppress cancer $(41,42)$. The present study clearly demonstrates that NK-92 cells inhibited the invasive phenotype of TNBC cells via the downregulation of uPA. Based on these findings, we propose that UPA may be a promising target for therapeutic strategies against TNBC.

\section{Acknowledgements}

Not applicable.

\section{Funding}

The present study was supported by the National Research Foundation of Korea (No. 2016R1A6A1A03007648 and 2019R1A2C1009773).

\section{Availability of data and materials}

The datasets used during the present study are available from the corresponding author upon reasonable request.

\section{Authors' contributions}

HJ, HJC, ESK and AM conceived and designed the study. HJ and HJC performed the experiments. HJ, HJC, ESK, HHL, HSC and AM analyzed and interpreted the data. HJ, HJC and AM contributed to the manuscript drafting and writing. HJ, HSC and AM reviewed and edited the manuscript. All authors read and approved the manuscript and agree to be accountable for all aspects of the research in ensuring that the accuracy or integrity of any part of the work are appropriately investigated and resolved. AM supervised the study.

\section{Ethics approval and consent to participate}

Not applicable.

\section{Patient consent for publication}

Not applicable.

\section{Competing interests}

The authors declare that they have no competing interests.

\section{References}

1. Bouchardy C, Fioretta G, Verkooijen HM, Vlastos G, Schaefer P, Delaloye JF, Neyroud-Caspar I, Balmer Majno S, Wespi Y, Forni M, et al: Recent increase of breast cancer incidence among women under the age of forty. Br J Cancer 96: 1743-1746, 2007.

2. Curado MP: Breast cancer in the world: Incidence and mortality. Salud Publica Mex 53: 372-384, 2011.

3. Redig AJ and McAllister SS: Breast cancer as a systemic disease: A view of metastasis. J Intern Med 274: 113-126, 2013.

4. Nakhjavani M, Hardingham JE, Palethorpe HM, Price TJ and Townsend AR: Druggable molecular targets for the treatment of triple negative breast cancer. J Breast Cancer 22: 341-361, 2019.

5. Bayraktar S and Glück S: Molecularly targeted therapies for metastatic triple-negative breast cancer. Breast Cancer Res Treat 138: 21-35, 2013

6. Kenny PA, Nelson CM and Bissell MJ: The Ecology of Tumors: By perturbing the microenvironment, wounds and infection may be key to tumor development. Scientist 20: 30, 2006.

7. Wang M, Zhao J, Zhang L, Wei F, Lian Y, Wu Y, Gong Z, Zhang S, Zhou J, Cao K, et al: Role of tumor microenvironment in tumorigenesis. J Cancer 8: 761-773, 2017.

8. Wu J and Lanier LL: Natural killer cells and cancer. Adv Cancer Res 90: 127-156, 2003.

9. Cheng M, Chen Y, Xiao W, Sun R and Tian Z: NK cell-based immunotherapy for malignant diseases. Cell Mol Immunol 10: 230-252, 2013.

10. Mandal A and Viswanathan C: Natural killer cells: In health and disease. Hematol Oncol Stem Cell Ther 8: 47-55, 2015.

11. López-Soto A, Gonzalez S, Smyth MJ and Galluzzi L: Control of metastasis by NK cells. Cancer Cell 32: 135-154, 2017.

12. Guillerey C, Huntington ND and Smyth MJ: Targeting natural killer cells in cancer immunotherapy. Nat Immunol 17: 1025-1036, 2016

13. Jiao Y, Huntington ND, Belz GT and Seillet C: Type 1 innate lymphoid cell biology: Lessons learnt from natural killer cells. Front Immunol 7: 426, 2016.

14. Sun Y, Yao Z, Zhao Z, Xiao H, Xia M, Zhu X, Jiang X and Sun C: Natural killer cells inhibit metastasis of ovarian carcinoma cells and show therapeutic effects in a murine model of ovarian cancer. Exp Ther Med 16: 1071-1078, 2018.

15. Aznavoorian S, Murphy AN, Stetler-Stevenson WG and Liotta LA: Molecular aspects of tumor cell invasion and metastasis. Cancer 71: 1368-1383, 1993.

16. Stamenkovic I: Matrix metalloproteinases in tumor invasion and metastasis. Semin Cancer Biol 10: 415-433, 2000.

17. Hanahan D and Weinberg RA: Hallmarks of cancer: The next generation. Cell 144: 646-674, 2011.

18. Song H, Ki SH, Kim SG and Moon A: Activating transcription factor 2 mediates matrix metalloproteinase-2 transcriptional activation induced by p38 in breast epithelial cells. Cancer Res 66: 10487-10496, 2006.

19. Lee S, Lee E, Ko E, Ham M, Lee HM, Kim ES, Koh M, Lim HK, Jung J, Park SY and Moon A: Tumor-associated macrophages secrete CCL2 and induce the invasive phenotype of human breast epithelial cells through upregulation of ERO1- $\alpha$ and MMP-9. Cancer Lett 437: 25-34, 2018.

20. Pepper MS: Role of the matrix metalloproteinase and plasminogen activator-plasmin systems in angiogenesis. Arterioscler Thromb Vasc Biol 21: 1104-1117, 2001.

21. Kumari S and Malla R: New insight on the role of plasminogen receptor in cancer progression. Cancer Growth Metastasis 8: 35-42, 2015.

22. Lijnen HR: Matrix metalloproteinases and cellular fibrinolytic activity. Biochemistry (Mosc) 67: 92-98, 2002.

23. Wilkins-Port CE, Higgins SP, Higgins CE, Kobori-Hotchkiss I and Higgins PJ: Complex regulation of the pericellular proteolytic microenvironment during tumor progression and wound repair: Functional interactions between the serine protease and matrix metalloproteinase cascades. Biochem Res Int 2012: 454368, 2012. 
24. Holst-Hansen C, Johannessen B, Høyer-Hansen G, Rømer J, Ellis $\mathrm{V}$ and Brünner $\mathrm{N}$ : Urokinase-type plasminogen activation in three human breast cancer cell lines correlates with their in vitro invasiveness. Clin Exp Metastasis 14: 297-307, 1996.

25. Crippa MP: Urokinase-ty pe plasminogen activator. Int J Biochem Cell Biol 39: 690-694, 2007.

26. Mauro CD, Pesapane A, Formisano L, Rosa R, D'Amato V, Ciciola P, Servetto A, Marciano R, Orsini RC, Monteleone F, et al: Urokinase-type plasminogen activator receptor (uPAR) expression enhances invasion and metastasis in RAS mutated tumors. Sci Rep 7: 9388, 2017.

27. Mahmood N, Mihalcioiu C and Rabbani SA: Multifaceted role of the urokinase-type plasminogen activator (uPA) and its receptor (uPAR): Diagnostic, prognostic, and therapeutic applications. Front Oncol 8: 24, 2018.

28. Moon A, Kim MS, Kim TG, Kim SH, Kim HE, Chen YQ and Kim HR: H-ras, but not N-ras, induces an invasive phenotype in human breast epithelial cells: A role for MMP-2 in the H-ras-induced invasive phenotype. Int J Cancer 85: 176-181, 2000.

29. Stepanova V, Jayaraman PS, Zaitsev SV, Lebedeva T, Bdeir K, Kershaw R, Holman KR, Parfyonova YV, Semina EV, Beloglazova IB, et al: Urokinase-type plasminogen activator (uPA) promotes angiogenesis by attenuating proline-rich homeodomain protein $(\mathrm{PRH})$ transcription factor activity and de-repressing vascular endothelial growth factor (VEGF) receptor expression. J Biol Chem 291: 15029-15045, 2016.

30. Tang Z, Li C, Kang B, Gao G, Li C and Zhang Z: GEPIA: A web server for cancer and normal gene expression profiling and interactive analyses. Nucleic Acids Res 45: W98-W102, 2017.

31. Lampelj M, Arko D, Cas-Sikosek N, Kavalar R, Ravnik M, Jezersek-Novakovic B, Dobnik S, Dovnik NF and Takac I: Urokinase plasminogen activator (uPA) and plasminogen activator inhibitor type-1 (PAI-1) in breast cancer-correlation with traditional prognostic factors. Radiol Oncol 49: 357-364, 2015.

32. Annecke K, Schmitt M, Euler U, Zerm M, Paepke D, Paepke S, von Minckwitz G, Thomssen C and Harbeck N: uPA and PAI-1 in breast cancer: Review of their clinical utility and current validation in the prospective NNBC-3 trial. Adv Clin Chem 45: $31-45,2008$.
33. Duffy MJ, McGowan PM, Harbeck N, Thomssen $\mathrm{C}$ and Schmitt M: uPA and PAI-1 as biomarkers in breast cancer: Validated for clinical use in level-of-evidence-1 studies. Breast Cancer Res 16: 428, 2014.

34. Fauriat C, Long EO, Ljunggren HG and Bryceson YT: Regulation of human NK-cell cytokine and chemokine production by target cell recognition. Blood 115: 2167-2176, 2010.

35. Sun W, Liu DB, Li W, Zhang LL, Long GX, Wang JF, Mei Q and $\mathrm{Hu}$ GQ: Interleukin-6 promotes the migration and invasion of nasopharyngeal carcinoma cell lines and upregulates the expression of MMP-2 and MMP-9. Int J Oncol 44: 1551-1560, 2014.

36. Razidlo GL, Burton KM and McNiven MA: Interleukin-6 promotes pancreatic cancer cell migration by rapidly activating the small GTPase CDC42. J Biol Chem 293: 11143-11153, 2018.

37. Gopinathan G, Milagre C, Pearce OM, Reynolds LE, Hodivala-Dilke K, Leinster DA, Zhong H, Hollingsworth RE, Thompson R, Whiteford JR and Balkwill F: Interleukin-6 stimulates defective angiogenesis. Cancer Res 75: 3098-3107, 2015.

38. Wegiel B, Bjartell A, Culig Z and Persson JL: Interleukin-6 activates PI3K/Akt pathway and regulates cyclin A1 to promote prostate cancer cell survival. Int J Cancer 122: 1521-1529, 2008.

39. Hsu CY, Bristow R, Cha MS, Wang BG, Ho CL, Kurman RJ, Wang TL and Shih IeM: Characterization of active mitogenactivated protein kinase in ovarian serous carcinomas. Clin Cancer Res 10: 6432-6436, 2004.

40. Leu CM, Wong FH, Chang C, Huang SF and Hu CP: Interleukin-6 acts as an antiapoptotic factor in human esophageal carcinoma cells through the activation of both STAT3 and mitogen-activated protein kinase pathways. Oncogene 22: 7809-7818, 2003.

41. Ulisse S, Baldini E, Sorrenti S and D'Armiento M: The urokinase plasminogen activator system: A target for anti-cancer therapy. Curr Cancer Drug Targets 9: 32-71, 2009.

42. Sidenius N and Blasi F: The urokinase plasminogen activator system in cancer: Recent advances and implication for prognosis and therapy. Cancer Metastasis Rev 22: 205-222, 2003. 